\title{
SIMPLE MODULES AND CENTRALIZERS $\left(^{(1}\right)$
}

\author{
BY \\ JOHN DAUNS
}

\begin{abstract}
A class of modules generalizing the simple ones is constructed. Submodule structure and centralizers of quotient modules are completely determined. The above class of modules is used to study the primitive ideal structure of the tensor products of algebras.
\end{abstract}

1. Introduction. A class of bimodules $M \equiv \boldsymbol{R}-M-\Delta$ (left $\boldsymbol{R}$, right $\Delta$ ) over rings or algebras $\boldsymbol{R}$ and $\Delta$ is constructed that generalizes simple left $\boldsymbol{R}$-modules, where for simple modules, $\Delta$ is the associated division ring. Consider algebras $\boldsymbol{A}_{\mathbf{1}}$ and $\boldsymbol{A}_{\mathbf{2}}$ over a field $F$ (of arbitrary characteristic). Unlike the simple modules, if $A_{i}-M_{i}-\Delta_{i}$ for $i=1,2$ are in the class, then so is also their tensor product, $A_{1} \otimes A_{2}-M_{1} \otimes M_{2}-\Delta_{1} \otimes \Delta_{2}$. Centralizers of quotient modules of $M_{1} \otimes M_{2}$ are determined in terms of $\Delta_{1} \otimes \Delta_{2}$. Due to being closed under tensor products, the above class is naturally suited for constructing simple $\boldsymbol{A} \otimes \boldsymbol{B}$-modules as quotient modules of $V \otimes W$ where $V, W$ are simple $\boldsymbol{A}, \boldsymbol{B}$-modules. Assume: (1) every $\boldsymbol{A}$-module is completely reducible (an identity is not assumed either for $\boldsymbol{A}$ or $\boldsymbol{B}$ ); (2) the centralizer of every simple $A$-module is $F$. Then every primitive ideal of $\boldsymbol{A} \otimes \boldsymbol{B}$ (over $F$ ) is uniquely of the form $\boldsymbol{A} \otimes P_{2}+P_{1} \otimes B$, where $P_{1} \subset A$ and $P_{2} \subset B$ are primitive.

2. Submodules and centralizers. Those parts of the theory in [7, pp. 111-113] that will be needed later are developed by using the techniques of [4] or of [8]. This way of approaching the subject is capable of slightly greater generality, and also sometimes is shorter.

Notation 2.1. Consider left modules $G$ and $N$ over any ring $\boldsymbol{R}$ (notation: $G=\boldsymbol{R}-G, N=\boldsymbol{R}-N$ and similarly for right modules). All rings considered here, such as $\boldsymbol{R}$, are not assumed necessarily to contain identities unless explicitly stated otherwise. The module $G$ generates $N$ if $N=\sum$ \{image $(\alpha) \mid \alpha \in \operatorname{Hom}_{R}(G, N)$; $G$ cogenerates $N$ if $(0)=\bigcap\left\{\operatorname{kernel}(\gamma) \mid \gamma \in \operatorname{Hom}_{R}(N, G)\right\}$. I.e. $G$ generates if $N$ is an epimorphic image of some direct sum of the $G$, while it cogenerates if $N$ can be embedded in a direct product of the $G$.

The centralizer $C(\boldsymbol{R}-M)$ of any module $\boldsymbol{R}-M$ is defined as $C(\boldsymbol{R}-M)$ $=\operatorname{Hom}_{R}(M, M)=$ End $M$, and it will be abbreviated as $C(M)$ or $C$ in case $R$ or

Received by the editors December 21, 1970 and, in revised form, August 26, 1971.

AMS 1970 subject classifications. Primary 16A64, 16A20; Secondary 16A20, 16A66.

Key words and phrases. Simple-module, division ring, endomorphism ring, centralizer, double centralizer, tensor product, primitive ideal.

( $\left.{ }^{1}\right)$ Research supported by National Science Foundation Grant GP-19824. 
$M$ are fixed. Then $M$ becomes a right $C$-module and an $\boldsymbol{R}-C$-bimodule. The notation $\boldsymbol{R}-M-C$ not only indicates that $M$ is a left $\boldsymbol{R}$ and a right $C$-module but also that the actions of $\boldsymbol{R}$ and $C$ commute. The double centralizer of $M$ is defined as $D(R-M)=C(M-C)$ and will be shortened to $D(M)=D$ if no confusion can arise.

Remarks 2.2. (1) There is a homomorphism $\lambda: R \rightarrow D(M)$.

(2) If $N=\oplus N(\alpha)$ is a direct sum of left $R$-modules and $p(\alpha): N \rightarrow N(\alpha)$ is the projection, then $p(\alpha) \in C(N)$ and $D N(\alpha) \subseteq N(\alpha)$. Consequently $D(\oplus N(\alpha)) \subseteq$ $\prod D(N(\alpha))$ is a subdirect product. Here and subsequently in direct sums the index set is understood and is omitted. Also, there are no restrictions on the cardinality of the index set.

(3) A similar result holds for products $N=\prod N(\alpha)$; the projection $p(\alpha): N$ $\rightarrow N(\alpha)$ again belongs to $C(N)$ and $D(N) \subseteq \prod D(N(\alpha))$ is a subdirect product.

2.3. Suppose $M=G \oplus N$, where $M, G$, and $N$ are left $R$-modules. Let $p: M \rightarrow G$ be the projection. View $G$ and $N$ as submodules of $M$. Frequently it is useful to view $C(G)$ as a subset of $C(M)$. Any $\gamma \in C(G)$ extends to $\gamma: G \oplus N$ $\rightarrow G \subset G \oplus N$-where the extended map is also called $\gamma$-by setting the restriction $\gamma \mid N=0$. Thus $\gamma \in C(M)$. The projection $p$ induces a ring homomorphism $\bar{p}$ by

$$
\bar{p}: D(G \oplus N) \rightarrow D(G), \quad \psi \rightarrow \psi^{*},
$$

where $\psi^{*}=p \psi p=\psi \mid G$ is the restriction and corestriction of $\psi$ to $G$. Alternatively, $D(G \oplus N) \subseteq D(G) \oplus D(N)$ and the above map is simply the projection onto the first summand $D(G)$. Thus there is a diagram

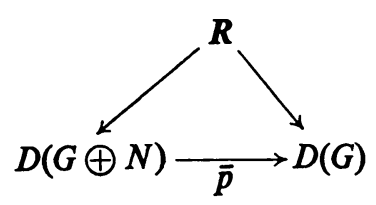

LemMA 2.4. If $M=G \oplus N$ and $\bar{p}$ are as above in 2.3 , then

(i) $\bar{p}$ is monic if $G$ generates or cogenerates $M$.

(ii) If $G$ generates and cogenerates, then $\bar{p}$ is an isomorphism.

Proof. Conclusion (i) is straightforward (see also [8, p. 430]). The proof of (ii) is omitted because it is given by K. Fuller in [4], except for a brief indication of how in [4] an element $b \in D(G)$ is extended to $D(G \oplus N)$. Since $G$ generates, each $v \in G \oplus N$ is of the form $v=\sum g k$ with $g \in G, k \in C(G)$. Then define $\beta v=\sum(b g) k$. Note that $\beta \mid G=b$.

The next corollary follows immediately from the preceding lemma. Alternatively, a direct proof can be found in [7, p. 112, Theorem 2].

Corollary 2.5. Assume $M=G \oplus N$ is a direct sum of left $R$-modules and that $G$ generates $M$. Then

$$
D(G)=\boldsymbol{R} \Rightarrow D(M)=\boldsymbol{R} .
$$


Definition 2.6. Suppose $M$ is a left module over two rings $\boldsymbol{R}$ and $D$. The ring $\boldsymbol{R}$ is said to be dense in $D$ provided any map between finite subsets of $M$ induced by an element $d \in D$ can be realized by an element from $\boldsymbol{R}$. That is, more precisely,

$\forall d \in D, \forall$ integer $k$ and $\forall m(1), \ldots, m(k) \in M$

$$
\exists r \in \boldsymbol{R} \text { such that } d m(j)=r m(j), \quad j=1, \ldots, k .
$$

REMARKS 2.7. (1) In particular, if $D \equiv D(\boldsymbol{R}-M)$ and if $\lambda: R \rightarrow D$ is the natural map with kernel $\{r \in R \mid r M=(0)\}$, then $\boldsymbol{R}$ is dense in $D$ provided $\lambda(\boldsymbol{R})$ is dense in the topological sense in

$$
\lambda(\boldsymbol{R}) \subseteq D \subset M^{M} \equiv \prod\{M \mid M\},
$$

when $M$ has the discrete topology.

(2) Any $D$-submodule of $M$ is also an $\boldsymbol{R}$-submodule. If the property in the above definition holds for $k=1$, then $D$ - and $\boldsymbol{R}$-submodules coincide.

Definition 2.8. Consider a direct sum of left $R$-modules $M=\oplus M(\alpha)$. Let $B=\|b(\alpha, \beta)\|$ be a (possibly infinite) matrix with entries $b(\alpha, \beta) \in \operatorname{Hom}_{R}(M(\alpha), M(\beta))$. Then $B$ is called row summable if

$\forall \alpha, \forall x \in M(\alpha), \quad x b(\alpha, \beta)=0$ for all but at most a finite number of $\beta$.

REMARK 2.9. In the above definition, $\operatorname{Hom}_{R}(M, M)$ consists precisely of all row summable matrices $B$, where each $x \in M$ can be written as a row vector

$$
x=x(\alpha(1))+\cdots+x(\alpha(n))=(0 \cdots 0, x(\alpha(1)), \ldots, x(\alpha(n)), 0 \cdots 0),
$$

where $x(\alpha(k)) \in M(\alpha(k))$ and $x B$ is the ordinary matrix product.

2.10. Suppose that $M$ decomposes as the direct sum $M=\oplus M(\alpha)$ of left $R$ modules each isomorphic to a fixed left $R$-module $G$ under $e(\alpha): G \rightarrow M(\alpha)$. Let $p(\alpha): M \rightarrow M(\alpha)$ be the projection and $i(\alpha): M(\alpha) \rightarrow M$ the inclusion map. Then $C(M)$ is isomorphic to the ring of column summable matrices with coefficients in $C(G)$ under the map

$$
c \in C(M), \quad c \rightarrow\|b(\alpha, \beta)\|,
$$

where $b(\alpha, \beta)=e(\alpha) i(\alpha) c p(\beta) e(\beta)^{-1} \in C(G)$.

NotAtion 2.11. For any module $M$ over any $\operatorname{ring} \boldsymbol{R}, \mathscr{L}(\boldsymbol{R}-M)$ denotes the lattice of left $\boldsymbol{R}$-submodules of $M$, and similarly for right modules. If $(C,+)$ is any ring $C$ viewed as a left module over itself, then $\mathscr{L}(C-(C,+))$ is the lattice of left ideals of $C$.

The next lemma (see [7, p. 111]) and corollary (see [7, p. 112] for a proof) will be used several times later.

Lemma 2.12. For $M=\oplus M(\alpha), e(\alpha): G \rightarrow M(\alpha)$, and $p(\alpha): M \rightarrow M(\alpha)$ as in 2.10 , there is a lattice isomorphism of the lattice of right $C(G)$-submodules of $G$ and right $C(M)$-submodules of $M$ given by

$$
\begin{aligned}
\mathscr{L}(G-C(G)) & \leftrightarrow \mathscr{L}(M-C(M)), \\
H & \rightarrow \bigoplus H e(\alpha), \\
N p(\alpha) e(\alpha)^{-1} & \leftarrow N,
\end{aligned}
$$

where the last inverse map is independent of the choice of index $\alpha$. 
CoROllary 2.13. Suppose $\Delta$ is a ring with $1 \in \Delta$ and $M=M-\Delta$ is a free right $\Delta$-module. There is a lattice isomorphism of the lattice of left ideals of $\Delta$ and the lattice of $C(M-\Delta)$ submodules of $M$ given by

$$
\begin{aligned}
\mathscr{L}(\Delta-(\Delta,+)) & \rightarrow \mathscr{L}(C(M-\Delta)-M), \\
L & \rightarrow M L .
\end{aligned}
$$

3. A class of modules. A class of modules similar but more general than the simple modules is constructed. Submodules, quotient modules, and tensor products of the modules of this class are investigated.

Notation 3.1. Suppose $\boldsymbol{R}$ is any ring $(1 \in \boldsymbol{R}$ or $1 \notin \boldsymbol{R})$ and $\Delta$ is a second ring with $1 \in \Delta$. Let $M$ be an $\boldsymbol{R}, \Delta$-bimodule with $\boldsymbol{R}$ acting on the left, $\Delta$ on the right. Assume $M-\Delta$ is free on a set $X=\{x(\alpha)\}$ of generators.

It should be noted that if the property described below holds for some particular integer $n$, then it also holds for all smaller integers.

3.2. For any integer $n$, the following three conditions (a), (b), and (c) are equivalent. For any distinct elements $x(1), \ldots, x(n) \in X$, there exists an $r \in \boldsymbol{R}$ with the following properties:

(a) $\forall z \in X, d \in \Delta, \exists r \in \boldsymbol{R}$ such that $r x(1)=z d, r x(2)=\cdots=r x(n)=0$.

(b) $\forall w \in M, \exists r \in R$ such that $r x(1)=w, r x(2)=\cdots=r x(n)=0$.

(c) $\forall w(1), \ldots, w(n) \in M, \exists r \in \boldsymbol{R}$ such that $r x(j)=w(j)$ for $j=1, \ldots, n$.

Proof. Since (c) $\Rightarrow($ b) $\Rightarrow$ (a), it will suffice to show that (a) $\Rightarrow$ (b) and (b) $\Rightarrow$ (c).

(a) $\Rightarrow(\mathrm{b})$. Write $w=z(1) d(1)+\cdots+z(p) d(p)$ for $z(i) \in\{x(\alpha)\}$, and $d(i) \in \Delta$.

By (a), there exists $r(j) \in \boldsymbol{R}$ with $r(j) x(1)=z(j) d(j), r(j) x(2)=\cdots=r(j) x(n)=0$.

Set $r=r(1)+\cdots+r(p)$. Then $r x(1)=w$ while $r x(2)=\cdots=r x(n)=0$.

(b) $\Rightarrow$ (c). By (b), there exist $r(j) \in \boldsymbol{R}$ with $r(j) x(j)=w(j), r(j) x(i)=0$ for $i \neq j$. Set $r=r(1)+\cdots+r(n)$. Then $r x(j)=r(j) x(j)=w(j)$. Thus (b) $\Rightarrow(\mathrm{c})$.

DEFINITION 3.3. If for all $n$ any one of the three equivalent conditions 2.2(a), (b), or (c) hold, then $\boldsymbol{R}$ will be said to satisfy the transitivity condition on $M$ with respect to $X$ and $\Delta$.

The class $\mathscr{K}$ consists of triples $(\boldsymbol{R}, M, \Delta)$ or bimodules $\boldsymbol{R}-M-\Delta$, where $\boldsymbol{R}$ is any ring $(1 \in \boldsymbol{R}$ or $1 \notin \boldsymbol{R}), \Delta$ is any ring with $1 \in \Delta$ such that

(1) $M-\Delta$ is free on a set $X \subset M$ of generators.

(2) Furthermore, $R-M-\Delta$ and $X$ satisfy the transitivity condition.

For any $\boldsymbol{R}-M-\Delta \in \mathscr{K}, \lambda$ denotes the natural map $\lambda: R \rightarrow C(M-\Delta)$.

Let $F$ be any field of arbitrary characteristic. Let $\mathscr{K} F \subset \mathscr{K}$ be the subclass of all $\boldsymbol{R}-M-\Delta \in \mathscr{K}$ such that $\boldsymbol{R}$ and $\Delta$ are algebras over $F$ and $M$ is an $F$-vector space and an $F$-algebra bimodule over $\boldsymbol{R}$ and $\Delta$.

Suppose $R-M-\Delta \in \mathscr{K}$ with respect to free generators $X$ of $M-\Delta$ and that $Y$ is any other set of free generators of $M-\Delta$. Then $X$ and $Y$ are linearly related by column finite matrices over $\Delta$ that are inverses of each other. As a consequence of the latter, it can be shown that the transitivity condition also holds with respect to $Y$. 
The next lemma gives a concrete way of constructing many examples of modules in the class $\mathscr{K}$.

LEMMA 3.4. If $\Delta$ is any ring with identity and $M-\Delta$ any free right module over $\Delta$, and if $\boldsymbol{R}$ is defined as $\boldsymbol{R}=C(M-\Delta)$, then

(i) $\boldsymbol{R}-M-\Delta \in \mathscr{K}$;

(ii) $C(\boldsymbol{R}-M)=\Delta$ and $D(\boldsymbol{R}-M)=\boldsymbol{R}$.

Proof. (i) Use of 2.10 for right modules shows that $\boldsymbol{R}$ is the ring of all row summable matrices with coefficients in $C((\Delta,+)-\Delta)=\Delta$. Thus 3.2(c) holds and (i) follows.

(ii) Note that $\Delta \subseteq C(R-M)$ because $\Delta$ acts faithfully on $M$. Since $M$ is right $\Delta$-free, it is of the form $M=\bigoplus x(\alpha) \Delta$, where each $x(\alpha) \Delta$ generates (and also cogenerates) $M$. Since $x(\alpha) \Delta \cong \Delta, D(x(\alpha) \Delta-\Delta)=\Delta$. Now apply the dual of Corollary 2.5 for right modules (with $G=x(\alpha) \Delta)$ to conclude that $D(M-\Delta)=\Delta$. But then $C(\boldsymbol{R}-M)=D(M-\Delta)=\Delta$. Finally, $D(\boldsymbol{R}-M)=C(M-\Delta)=\boldsymbol{R}$.

Proposition 3.5. For $\boldsymbol{R}-M-\Delta \in \mathscr{K}$,

(i) $R$ is dense in $C(M-\Delta)$; consequently the lattices of submodules of these two rings are equal, i.e. $\mathscr{L}(\boldsymbol{R}-M)=\mathscr{L}(C(M-\Delta)-M)$.

(ii) There is a lattice isomorphism of the lattice of left ideals of $\Delta$ onto the lattice of left $\boldsymbol{R}$-submodules of $M$ given by

$$
\mathscr{L}(\Delta-(\Delta,+)) \rightarrow \mathscr{L}(\boldsymbol{R}-M), \quad L \rightarrow M L .
$$

Proof. (i) For any $m(1), \ldots, m(k) \in M$, and any $s \in C(M-\Delta)$, there are a finite number $x(1), \ldots, x(n) \in X=\{x(\alpha)\}$-a $\Delta$-free basis of $M$-such that each $m(j)$ $=x(1) d(1, j)+\cdots+x(n) d(n, j)$, where $d(i, j) \in \Delta$ may be zero. By the transitivity condition $3.2(\mathrm{c})$ (with $w(j) \equiv s x(j)$ ), there exists an $r \in R$ with $r x(j)=s x(j)$ for $j=1, \ldots, n$. But then since both $r$ and $s$ commute with $\Delta$, also $r m(j)=\operatorname{sm}(j)$ for all $j$. Hence $\boldsymbol{R}$ is dense in $C(M-\Delta)$. Thus the $R$-submodules of $M$ coincide with the $C(M-\Delta)$-submodules. Conclusion (ii) follows from the assertion that $\mathscr{L}(\Delta-(\Delta,+))$ $\cong \mathscr{L}(C(M-\Delta)-M)$ in Corollary 2.13 .

The first part (i) of the next theorem is established in slightly greater generality than the present setting of class $\mathscr{K}$.

3.6. THEOREM I. Let $\boldsymbol{R}$ and $\Delta$ be any rings (with or without identities) and $M=R-M-\Delta$ any $\boldsymbol{R}-\Delta$-bimodule. Assume that $M \Delta=M$. Let $L \subset \Delta$ be any left ideal and form the left $R$-module $M / M L$. Then

(i) there is a natural homomorphism $C(\Delta-\Delta / L) \rightarrow C(R-M / M L)$.

Now assume in addition that $\boldsymbol{R}-M-\Delta \in \mathscr{K}$, i.e. that

(1) $1 \in \Delta$ and $M-\Delta$ is $\Delta$-free;

(2) $\boldsymbol{R}$ acts transitively (see 2.2) on a set of free generators of $M-\Delta$.

Then the map in (i) is

(ii) monic if (1) holds;

(iii) and a bijective isomorphism if both (1) and (2) hold. 
Proof. If $\Delta$ is any ring $(1 \in \Delta$ or $1 \notin \Delta)$ and $L \subset \Delta$ is any regular left ideal, then by [7, p. 25] there is a ring isomorphism $C(\Delta-\Delta / L) \cong N(L) / K$, where $N(L)$ is the idealizer or normalizer $N(L) \equiv\{b \in \Delta \mid L b \subseteq L\}$ of $L$ in $\Delta$ and where $K$ $\equiv\{k \in \Delta \mid \Delta k \subseteq L\}$. Note that $L \subseteq K$ and $K$ is an ideal in $N(L)$. If $1 \in \Delta$ then $K=L$. Furthermore $\gamma=b+K \in N(L) / K$ acts on $\Delta / L$ by $(d+L) \gamma=d b+L$ for $d \in \Delta$.

(i) For $\gamma=b+K \in C(\Delta-\Delta / L)$, define $\gamma^{*} \in C(R-M / M L)$ by $(m+M L) \gamma^{*}=m b$ $+M L$. The map $\gamma \rightarrow \gamma^{*}$ is well defined, for if $b+K=c+K \in N(L) / K$, then $m \in M$ $=M \Delta$ and $\Delta K \subseteq L$ imply that $m(b-c) \in M \Delta(b-c) \subseteq M L$. The verification that $\gamma \rightarrow \gamma^{*}$ is a ring homomorphism is straightforward and is omitted.

(ii) Now assume (1). Let $X=\{x(\alpha)\}$ be a right $\Delta$-free basis of $M$. If the map $\gamma \rightarrow \gamma^{*}$ is not monic, then there are $b+L \neq b+L \in N(L) / L$, but with $m b+M L$ $=m \bar{b}+M L$ for all $m \in M$. Equivalently, $x(\alpha) c b+M L=x(\alpha) c \bar{b}+M L$ holds for all $x(\alpha)$ and all $c \in \Delta$. Pick any $x \in\{x(\alpha)\}$. Then

$$
x b-x \bar{b}=x l+x(1) l(1)+\cdots+x(k) l(k) \in M L=\oplus x(\alpha) L,
$$

where $l, 0 \neq l(i) \in L$ and where $x, x(1), \ldots, x(k) \in\{x(\alpha)\}$ are all distinct. This implies that $x(i) l(i)=0$ for all $i$ and that $x b-x \bar{b}=x l$. The freeness of $M$ over $\Delta$ implies that $b-\bar{b}=l \in L$ and that $b+L=\bar{b}+L$. Thus the map is monic.

Now assume (1) and (2). In order to show that $\gamma \rightarrow \gamma^{*}$ is epic, take any $\theta \in C(\boldsymbol{R}-M / M L)$. First it will be shown that for any member $x$ of any free $\Delta$ basis $\{x(\alpha)\}$ of $M-\Delta$, the map $\theta$ preserves "lines" in the sense that $(x \Delta+M L) \theta$ $\subseteq x \Delta+M L$. For any $d \in \Delta$, write $(x d+M L) \theta=x \bar{d}+z+M L$ where $z=x(1) d(1)$ $+\cdots+x(n) d(n)(d, 0 \neq d(i) \in \Delta)$, where $x, x(1), \ldots, x(n) \in\{x(\alpha)\}$ are all distinct. By assumption (2), there exists $r \in \boldsymbol{R}$ such that $r x=x, r x(1)=\cdots=r x(n)=0$. But then comparison of the two equations

$$
\begin{aligned}
& {[r(x d+M L)] \theta=[x d+M L] \theta=x \bar{d}+z+M L,} \\
& r[(x d+M L) \theta]=r[x \bar{d}+z+M L]=x \bar{d}+M L
\end{aligned}
$$

shows that $z \in M L$ and that $(x \Delta+M L) \theta \subseteq x \Delta+M L$.

It will be shown that for $\theta \in C(R-M / M L)$ there exists $\gamma \in C(\Delta-\Delta / L)$ with $\gamma^{*}=\theta$. For any $d+L \in \Delta / L,(d+L) \gamma$ is defined in three steps as follows:

(1) Pick any member $x$ of any free $\Delta$-basis of $M$.

(2) Define $\bar{d} \in \Delta$ by $(x d+M L) \theta=x \bar{d}+M L$.

(3) Set $(d+L) \gamma=\bar{d}+L$.

Thus far $\gamma: \Delta / L \rightarrow \Delta / L$ is an abelian group homomorphism. It remains to show that $[c d+L] \gamma=c[(d+L) \gamma]=c d+L$ for any $c \in \Delta$. There exists $r \in R$ such that $r x=x c$.

Since $\theta$ commutes with $r, \quad[r(x d+M L)] \theta=[x c d+M L] \theta=r[(x d+M L) \theta]$ $=r[x \bar{d}+M L]=x c \bar{d}+M L$. Use of the definition of $\gamma$ in the last equation shows that $(c d+L) \gamma=c \bar{d}+L=c[\bar{d}+L]=c[(d+L) \gamma]$. Thus $\gamma \in C(\Delta-\Delta / L) \cong N(L) / L$. However, now it follows further that $\gamma$ is of the form $\gamma=b+L$; where $b \in N(L)$ and $(d+L) \gamma$ $=d b+L$.

In order to conclude that $\gamma^{*}=\theta$, it will be necessary to show that the definition of 
$\gamma$ is independent of the choice of $x$. If $y$ is a member of even possibly some other free $\Delta$-basis of $M$, then simply choose $r \in R$ with $r x=y$. Then $r[(x d+M L) \theta]$ $=r[x \bar{d}+M L]=y \bar{d}+M L$, while $[r x d+M L] \theta=(y d+M L) \theta$.

By taking right $\Delta$-linear combinations of the members of any basis of $M$, such as $\{x(\alpha)\}$, it now follows that for any $m \in M,(m+M L) \theta=m b+M L$. Thus $\gamma^{*}=\theta$ and the two natural maps are inverses of one another, and $C(\Delta-\Delta / L)$ $\cong C(R-M / M L)$.

A corollary of the proof of the above theorem shows that $C(R-M / M L)$ preserves "lines" in $M / M L$.

3.7. Corollary to TheOREm I. Under the assumptions (1) and (2) of the last theorem, $(m+M L) C(\boldsymbol{R}-M / M L) \subseteq m \Delta+M L$ for any $m \in M$.

The next proposition describes the action of $R$ and $C(R-M / M L)$ on $M / M L$ much more concretely than the previous theorem. A curious by-product of the next proposition is that $M / M L$ can also be made into a left $\Delta$-module in a nonnatural nonunique way.

Proposition 3.8. Consider $\boldsymbol{R}-M-\Delta \in \mathscr{K}$, with $M-\Delta$ free on $X=\{x(\alpha) \mid \alpha \in J\}$, where $J$ is an indexing set, and any left ideal $L \subset \Delta$. Define $\Gamma$ to be $\Gamma=C(R-M / M L)$. Then the module $M / M L$ has the following properties:

(i) If $\bar{x}(\alpha)$ is defined as $\bar{x}(\alpha)=1+L \in \Delta / L$ for each $\alpha \in J$, then there is an isomorphism of right $\Gamma$-modules

$$
\begin{aligned}
M / M L & \rightarrow \oplus\{\bar{x}(\alpha) \Delta / L \mid \alpha \in J\}, \\
x(\alpha) t+M L & \rightarrow \bar{x}(\alpha)(t+L), \quad t \in \Delta .
\end{aligned}
$$

(ii) $\boldsymbol{R}$ acts on $M / M L$ as follows: for any $r \in R, x(\alpha) \in X$, and $\eta \in \Delta / L$, if $r x(\alpha)$ $=x(1) d(1)+\cdots+x(n) d(n)$ for $x(i) \in X$ and $d(i) \in \Delta$, then

$$
r[\bar{x}(\alpha) \eta]=\sum_{i=1}^{n} \bar{x}(i) d(i) \eta .
$$

(iii) $\Gamma$ acts on $M / M L$ via the isomorphism $\Gamma \cong C(\Delta-\Delta / L)$ and by respecting the direct sum decomposition in (i).

(iv) If $L$ is an ideal in $\Delta$, then $\Gamma \cong C(\Delta-\Delta / L)=\Delta / L$ and $R-M / M L-\Gamma \in \mathscr{K}$.

Proof. Conclusions (i), (ii), and (iii) follow from the previous theorem, and from the isomorphism

$$
M / M L=\oplus x(\alpha) \Delta / \oplus x(\alpha) L \cong \oplus \bar{x}(\alpha)(\Delta / L) .
$$

For any left ideal, if $1 \in \Delta, C(\Delta-\Delta / L)=N(L) / L$, where $N(L)=\{b \in \Delta \mid L b \subseteq L\}$. If $L$ is an ideal, then $N(L)=\Delta$. But then by (i), $M / M L-\Gamma$ is free on $\{\bar{x}(\alpha) \mid \alpha \in J\}$ and, clearly, the transitivity requirement carries over.

4. Tensor products. A shortcoming of the class of simple modules is that they are not closed under tensor products. This defect is remedied by the class $\mathscr{K} F$. 
First, a brief explanation is given why tensor products of simple modules need not be simple.

Definition 4.1. Let $A$ be any algebra over any field $F$ of arbitrary characteristic. ( $1 \in A$ is not assumed.) Suppose $V=A-V$ is any left $\boldsymbol{A}$-algebra module. For an integer $n, A$ is $n$-transitive on $V$ (with respect to $F$ ) provided for any $F$-linearly independent elements $x(1), \ldots, x(n) \in V$ and any arbitrary set of elements $w(1), \ldots, w(n) \in V$, there exists an element $a \in A$ with $a x(i)=w(i)$ for $i=1, \ldots, n$. The module $A-V$ is simple if there are no proper $A$-submodules and if $A V=V$ $\neq(0)$. It is absolutely simple if $C(A-V)=F$.

Let $M(A)$ be the algebra of left and right translations by $A$ acting on the vector space $(A,+)$ and view $A$ as a left $M(A)$-module. Then $A$ is simple if $M(A)-(A,+)$ is simple, $A$ is central simple if $M(A)-(A,+)$ is absolutely simple (see [7, p. 109]).

The next remarks will clarify the subsequent theorems.

REMARKS 4.2. The following facts for an $\boldsymbol{A}$-module $V$ are either trivial, or are folklore, or follow immediately from the previous considerations.

(1) $V$ is simple if and only if $\boldsymbol{A}$ is 1-transitive.

(2) $V$ is absolutely simple if and only if $\boldsymbol{A}$ is $n$-transitive for every $n$.

(3) $n$-transitivity is equivalent to 2-transitivity.

(4) Assume that $F$ is algebraically closed and that the dimension of $V$ over $F$ is strictly smaller than the cardinality of $F$. If $V$ is simple, then $C(A-V)=F$ and $V$ is absolutely simple.

(5) Consider simple left modules $M_{1}$ and $M_{2}$ over algebras $A_{1}$ and $A_{2}$. Abbreviate "simple" by "s", "central simple" by "c.s", and "absolutely simple" by "ab.s" Then $M_{1} \otimes M_{2}$ has the following properties:

\begin{tabular}{c|c|c}
$M_{1}$ & $M_{2}$ & $M_{1} \otimes M_{2}$ \\
\hline $\mathrm{s}$ & $\mathrm{s}$ & $?$ \\
\hline $\mathrm{s}$ & $\mathrm{ab} . \mathrm{s}$ & $\mathrm{s}$ \\
\hline $\mathrm{ab} . \mathrm{s}$ & $\mathrm{ab} . \mathrm{s}$ & $\mathrm{ab} . \mathrm{s}$
\end{tabular}

If $A_{1}$ and $A_{2}$ are simple, then in particular, the above may be applied to $\left(\boldsymbol{A}_{i},+\right)$ as a left $M\left(A_{i}\right)$-module (see [7, p. 114]):

\begin{tabular}{c|c|c}
$A_{1}$ & $A_{2}$ & $A_{1} \otimes A_{2}$ \\
\hline $\mathrm{s}$ & $\mathrm{s}$ & $?$ \\
\hline $\mathrm{s}$ & $\mathrm{c.s}$ & $\mathrm{s}$ \\
\hline $\mathrm{c} . \mathrm{s}$ & $\mathrm{c} . \mathrm{s}$ & $\mathrm{c.s}$
\end{tabular}

Conclusions (iii) and (iv) in the theorem below are generalizations of the 
Azumaya-Nakayama Theorem ([7, p. 114, Theorem 1]), which only deals with simple modules.

4.3. Theorem II. Suppose $A_{i}-M_{i}-\Delta_{i} \in \mathscr{K} F$ (see 3.3) for $i=1$, 2. Suppose $X=\{x(\alpha)\}, Y=\{y(\beta)\}$ are the $\Delta_{1}, \Delta_{2}$-free bases of $M_{1}, M_{2}$. Define $A-M-\Delta$ and $X \otimes Y$ as $A=A_{1} \otimes A_{2}, M=M_{1} \otimes M_{2}, \Delta=\Delta_{1} \otimes \Delta_{2}$, and $X \otimes Y=\{x(\alpha) \otimes y(\beta)\}$.

(i) Then $M-\Delta$ is free on $X \otimes Y$.

(ii) $A$ acts transitively on $M$ (see 3.2) and thus $A-M-\Delta \in \mathscr{K} F$.

(iii) If $C\left(A_{i}-M_{i}\right)=\Delta_{i}$ for $i=1$ and 2 , then $C(A-M)=\Delta$.

(iv) The lattice of left $A$-submodules of $M$ is isomorphic to the lattice of left ideals of $\Delta$ under

$$
\mathscr{L}(\Delta-(\Delta,+)) \rightarrow \mathscr{L}(A-M), \quad L \rightarrow M L
$$

Proof. (i) Since the tensor product commutes with direct sums, it follows from $M_{1}=\bigoplus x(\alpha) \Delta_{1}$ and $M_{2}=\oplus y(\beta) \Delta_{2}$ that $M=\oplus x(\alpha) \otimes y(\beta) \Delta_{1} \otimes \Delta_{2}$ is a free $\Delta$ module.

(ii) It will be shown that the criterion 3.2(a) holds. Given an integer $n$, an element $d \in \Delta$, and $z, z(1), \ldots, z(n) \in X \otimes Y$ with $z(i)=x(\alpha(i)) \otimes y(\beta(i))$, where $(\alpha(i), \beta(i))$ $=(\alpha(j), \beta(j))$ if and only if $i=j$. Set $x(i)=x(\alpha(i)), y(j)=y(\beta(j))$ and list all $x(i), y(j)$ appearing in the $z(k)$ 's:

$$
x(1), \ldots, x(p) ; \quad y(1), \ldots, y(q) .
$$

Then $\{z(1), \ldots, z(n)\} \subseteq\{x(i) \otimes y(j) \mid 1 \leqq i \leqq p ; i \leqq j \leqq q\}$. It suffices to find an $r \in A$ such that $r x(1) \otimes y(1)=z d$ and $r x(i) \otimes y(j)=0$ for $(i, j) \neq(1,1)$. Write

$$
\begin{aligned}
z & =x \otimes y, \quad x \in X, y \in Y \\
d & =d(1) \otimes \delta(1)+\cdots+d(t) \otimes \delta(t), \quad d(i) \in \Delta_{1}, \delta(i) \in \Delta_{2} ; \\
z d & =x d(1) \otimes y \delta(1)+\cdots+x d(t) \otimes y \delta(t) .
\end{aligned}
$$

For each $k=1, \ldots, t$ there exist elements satisfying

$$
\begin{array}{lll}
a(k) \in A_{1}: a(k) x(1)=x d(k), & a(k) x(i)=0 & \text { for } i \neq 1 \\
b(k) \in A_{2}: b(k) y(1)=y \delta(k), & b(k) y(j)=0 & \text { for } j \neq 1 .
\end{array}
$$

Finally, if $r$ is defined as $r=a(1) \otimes b(1)+\cdots+a(t) \otimes b(t)$, then

$$
r x(1) \otimes y(1)=z d, \quad r x(i) \otimes y(j)=0 \quad \text { if }(i, j) \neq(1,1) .
$$

(iii) Set $\Gamma=C(M-\Delta)$. Then, first, since $M-\Delta$ is free, $D(M-\Delta)=C(\Gamma-M)$ $=\Delta$. By 3.5(i), $A$ is dense in $\Gamma$; hence $C(A-M) \subseteq C(\Gamma-M)$. Since $\Delta=C\left(A_{1}-M_{1}\right)$ $\otimes C\left(A_{2}-M_{2}\right), \Delta \subseteq C(A-M)$. Combining the two inclusions, we get $\Delta \subseteq C(A-M)$ $\subseteq C(\Gamma-M)=\Delta$, and thus $C(A-M)=\Delta$.

(iv) The proof of (iv) follows immediately from 3.5.

It is not clear under what hypotheses the next proposition can be generalized from ideals to subrings. 
Proposition 4.4. Consider a module $M=\boldsymbol{R}-M$ over any ring $\boldsymbol{R}$. Suppose $I \subset \boldsymbol{R}$ is any ideal such that, for any $m \in M, I m=(0) \Rightarrow m=0$. (In particular, if $\boldsymbol{R}-M$ is simple and $I M \neq(0)$, then for any $0 \neq m \in M, I m=M$ and the above applies.) Then $C(R-M)=C(I-M)$.

Proof. Even for a subring $I \subset \boldsymbol{R}, C(\boldsymbol{R}-M) \subseteq C(I-M)$ always holds. Suppose $m=(r v) \gamma-r(v \gamma)$ for $r \in R, v \in M$, and $\gamma \in C(I-M)$. Then for any $i \in I$,

$$
i m=i[(r v) \gamma]-(i r)(v \gamma)=[i(r v)] \gamma-[(i r) v] \gamma=0 .
$$

Thus $m=0, \gamma \in C(R-M)$, and $C(R-M)=C(I-M)$.

REMARK 4.5. The proof of the next observation is elementary and is omitted. Suppose $\boldsymbol{A}_{\boldsymbol{i}}-\boldsymbol{M}_{\boldsymbol{i}}-\Delta_{\boldsymbol{i}}$ and $\boldsymbol{A}-M-\Delta \in \mathscr{K} F$ as in Theorem II. Define $\boldsymbol{A}_{\boldsymbol{i}}{ }^{\mathbf{1}}=\boldsymbol{A}_{\boldsymbol{i}}$ if $1 \in A_{i}$ or adjoin an identity in the usual way if $1 \notin A_{i}$ by forming $F \times A_{i}$. Then

$$
\begin{aligned}
C(A-M)=C\left(A_{1}{ }^{1} \otimes A_{2}-M\right) & =C\left(A_{1}{ }^{1} \otimes A_{2}{ }^{1}-M\right), \\
& A_{1}{ }^{1} \otimes A_{2}-M-\Delta \text { and } A_{1}{ }^{1} \otimes A_{2}{ }^{1}-M-\Delta \in \mathscr{K} F .
\end{aligned}
$$

Conclusion (iii) in the next theorem in case both $M_{1}$ and $M_{2}$ are simple modules is proved in [6]. In case $A_{1}$ and $A_{2}$ are primitive, there do not seem to be necessary and sufficient conditions for $A_{1} \otimes A_{2}$ to be primitive (see [6, p. 20, Theorem 1]).

4.6. THEOREM III. In addition to the hypotheses of Theorem 4.3, assume that

(a) $M_{1}$ and $M_{2}$ are faithful left $A_{1}$ - and $A_{2}$-modules; one of the modules $A_{1}-M_{1}$ or $\mathrm{A}_{\mathbf{2}}-\mathrm{M}_{\mathbf{2}}$ is cyclic.

(b) $L \subset \Delta$ is a left ideal such that $L: \Delta=\{\eta \in \Delta \mid \eta \Delta \subseteq L\}=(0)$.

Then the following hold:

(i) $M / M L$ is a faithful A-module.

(ii) Consequently, by taking $L=\{0\}$, it follows that in particular $M$ is a faithful A-module.

(iii) If $\Delta$ is a left primitive ring, i.e. if $(0)=L: \Delta$ for some maximal left ideal $L$, then $M / M L$ is a simple faithful A-module.

Proof. Let a nonzero element

$$
\xi=\sum_{i=1}^{n} a(i) \otimes b(i) \in A_{1} \otimes A_{2}
$$

be given with $b(1), \ldots, b(n)$ linearly independent. Suppose $\{x(\alpha)\},\{y(\beta)\}$ are the free $\Delta_{1}, \Delta_{2}$-bases of $M_{1}, M_{2}$. Assume $M_{2}=A_{2} w$ is cyclic where $w \in M_{2}$. For any $x(\alpha)$, there are $c(k, i) \in \Delta_{1}$ and $d(k, i) \in \Delta_{2}$ such that

$$
\begin{aligned}
a(i) x(\alpha) & =x(1) c(1, i)+\cdots+x(p) c(p, i), \\
b(i) w & =y(1) d(1, i)+\cdots+y(q) d(q, i),
\end{aligned}
$$

with $x(1), \ldots, x(p) \in\{x(\alpha)\}$ and $y(1), \ldots, y(q) \in\{y(\beta)\}$ all distinct. By allowing some of the $c(k, i)$ and $d(k, i)$ to be zero we may assume that $p, q$, the $x(k)$, and 
$y(k)$ are the same for all $i$. Since $a(1) M_{1} \neq 0$, there is an $\alpha$ with $a(1) x(\alpha) \neq 0$, and the notation may so be chosen that $c(1,1) \neq 0$. By the transitivity condition, there exists an $a \in A_{1}$ with $a x(1)=x(1), a x(2)=\cdots=a x(p)=0$. Set $c(i)=c(1, i)$. If $1 \notin \boldsymbol{A}_{2}$, form $\boldsymbol{A}_{\mathbf{2}}{ }^{1}$ and consider $\boldsymbol{A}_{\mathbf{2}}{ }^{1}-\boldsymbol{M}_{\mathbf{2}}$. A computation shows that

$$
(a \otimes 1) \xi x(\alpha) \otimes w=\sum_{i=1}^{n} x(1) c(i) \otimes b(i) w .
$$

Since $A_{2}-M_{2}$ is faithful and since the $b(i)$ are linearly independent, also $b(1) w$, $\ldots, b(n) w$ are linearly independent. But then $M_{1} \otimes b(1) w, \ldots, M_{1} \otimes b(n) w$ are $n$ linearly independent subspaces. Thus the sum (1) is not zero because the nonzero elements of $\{x(1) c(i) \otimes b(i) w\}$ are linearly independent and $x(1) c(1) \otimes b(1) w \neq 0$. Thus also $\xi x(\alpha) \otimes w \neq 0$ and (ii) $A$ acts faithfully on $M$. Equation (1) can be rewritten as

$$
(a \otimes 1) \xi x(\alpha) \otimes w=\sum_{k=1}^{q} x(1) \otimes y(k) \sum_{i=1}^{n} c(i) \otimes d(k, i) \neq 0 .
$$

Thus at least one of the $q$ summands in the above sum on $k$ is not zero. Choose the notation so that this nonzero term occurs for $k=1$; set $d(i)=d(1, i)$ and $\eta=c(1) \otimes d(1)+\cdots+c(n) \otimes d(n)$. Thus $x(1) \otimes y(1) \eta \neq 0$. By transitivity, there is a $b \in A_{2}$ with $b y(1)=y(1), b y(2)=\cdots=b y(q)=0$. For any $t \in \Delta=\Delta_{1} \otimes \Delta_{2}$, equation (2) yields

$$
a \otimes b \xi[(x(\alpha) \otimes w) t]=x(1) \otimes y(1) \eta t .
$$

Due to the fact that $M-\Delta$ is free on $\{x(\alpha) \otimes y(\beta)\}$, it follows that

$$
x(1) \otimes y(1) \eta t \in M L \Leftrightarrow \eta t \in L .
$$

Since $\eta \neq 0$, hypothesis (b) guarantees that there exists $t \in \Delta$ with $\eta t \notin L$. Consequently $A-M / M L$ is faithful and (i) holds.

(iii) Since $M \in \mathscr{K}$, we have $\mathscr{L}(A-M / M L) \cong \mathscr{L}(\Delta-\Delta / L)$ by 3.5 (ii) and thus $M / M L$ is simple if and only if $L$ is a maximal left ideal. If $\Delta$ is primitive, $L$ may be so chosen that $M / M L$ is not only simple but also a faithful $\boldsymbol{A}$-module.

In order to illustrate the power and usefulness of Theorems I and III, a result of Harada and Kanzaki [6, pp. 19-20, Theorem 1(1)] is obtained immediately as a corollary. Due to the fact that in their formulation both a ring as well as its centralizer act on the same side of the module, they assumed $\boldsymbol{A}$ is right primitive, $\Delta$ left primitive; while the centralizers of $\Delta$ and $\boldsymbol{A}$-modules are anti-isomorphic. The present formulation is slightly simpler in that only left primitive rings and only isomorphisms need be considered.

4.7. Corollary to Theorems I AND III. Suppose $A_{i}$ are primitive algebras over any field $F$ with simple, faithful modules $M_{i}$ and associated division rings $\Delta_{i}$ $=C\left(A_{i}-M_{i}\right)$ for $i=1,2$. As before, if $A=A_{1} \otimes A_{2}$ and $\Delta=\Delta_{1} \otimes \Delta_{2}$, then

(i) $\Delta$ left primitive $\Rightarrow A$ is left primitive. 
(ii) For any simple faithful left $\Delta$-module $V$, there is a simple faithful left $A$ module $\bar{V}$.

(iii) Furthermore, $C(\Delta-V) \cong C(A-\bar{V})$.

Proof. Any simple, faithful $\Delta-V$ is of the form $V \cong \Delta / L$ where $L \subset \Delta$ is a maximal left ideal with

$$
L: \Delta=\{\eta \in \Delta \mid \eta \Delta \subseteq L\}=\{0\},
$$

and conversely. Set $M=M_{1} \otimes M_{2}$. Thus by Theorem 4.6, $A-M / M L$ is simple and faithful. Thus (i) and (ii) hold with $\bar{V}=M / M L$. (iii) By Theorem 4.3(iii), $C(A-M)$ $=\Delta$. But then Theorem 3.6 is applicable to $A-M-\Delta \in \mathscr{K}$, to conclude that the following division rings are naturally isomorphic:

$$
C(\Delta-\Delta / L) \cong C(A-M)
$$

5. Primitive ideals in tensor products. Throughout none of the algebras are assumed to have an identity unless otherwise stated.

Notation 5.1. If not stated otherwise, the term "module" refers to a left module. Also, a module $V$ over an algebra $\boldsymbol{A}$ is an algebra module. The endomorphism ring $\operatorname{Hom}_{A}(V, V)$ of $V$ is denoted by $D \equiv$ End $V$, and as before $D$ acts on the right of $V$. A module is completely reducible if it is a direct sum of simple modules.

Consider algebras $\boldsymbol{A}, \boldsymbol{B}$, and $E$ over a field $F$ (of arbitrary characteristic). As is customary for semigroups, for any algebra $A$, an algebra $A^{\mathbf{1}}$ is defined as $A^{\mathbf{1}}$ $=\boldsymbol{A}$ if $1 \in \boldsymbol{A}$ and $\boldsymbol{A}^{\mathbf{1}}=F \times \boldsymbol{A}$ otherwise with the usual operations $((f, a)(\bar{f}, \bar{a})$ $=(f \bar{f}, f \bar{a}+\bar{f} a+a \bar{a}))$. The reader may wish to simply assume $\boldsymbol{A}^{\mathbf{1}}=\boldsymbol{A}$ throughout. The symbol " $\triangleleft$ " denotes ideals, e.g. $\boldsymbol{A} \triangleleft \boldsymbol{A}^{\mathbf{1}}$. Any $\boldsymbol{A}$-module is an $\boldsymbol{A}^{\mathbf{1}}$-module if $1 \notin A$, and any algebra homomorphism $\phi: A \rightarrow E$ has a natural extension

$$
\phi: A^{1} \rightarrow F \times E, \quad(f, a) \rightarrow(f, \phi(a)),
$$

even when $1 \in E$. Abbreviate $1=(1,0) \in A^{1}$ and write $F(1,0) \otimes B=1 \otimes$ $B \subset A^{1} \otimes B$. All tensor products are over $F$.

5.2. Suppose $\phi: A \rightarrow E$ and $\psi: B \rightarrow E$ are homomorphisms whose images in $E$ commute elementwise. Then there is a unique map $\phi \otimes \psi$ and a commutative diagram

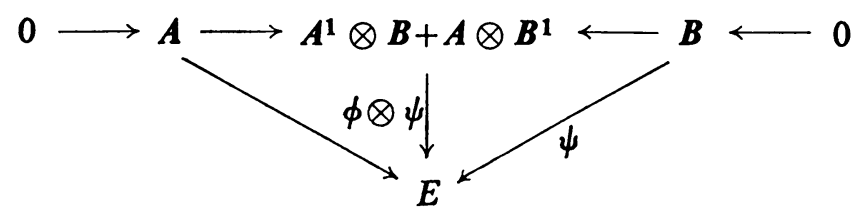

where $(\phi \otimes \psi)(a \otimes b)=\phi(a) \psi(b),(\phi \otimes \psi)(a \otimes 1)=\phi(a)$ and $(\phi \otimes \psi)(1 \otimes b)=\psi(b)$. The point of all of the above is that in general $\boldsymbol{A} \otimes \boldsymbol{B}$ does not have the universal property, while $(F \times \boldsymbol{A}) \otimes \boldsymbol{B}+\boldsymbol{A} \otimes(F \times \boldsymbol{B})$ is the unique tensor product in the category 
of all rings with or without identity and homomorphisms that need not preserve identities.

Recall that an exact category is one having kernels and cokernels, or equivalently, unique monic epic factorization. The next lemma is valid in any exact category in which the tensor product is an exact functor, and in particular for tensor products over a field. Its proof is a straightforward categorical argument and is omitted.

Lemma 5.3. Consider F-algebras $A, B$ and any left ideals $L_{1} \subset A, L_{2} \subset B$. Define $L=A \otimes L_{2}+L_{1} \otimes B$. Let $J \subset A \otimes B$ denote the left ideal defined as the kernel of the map $\boldsymbol{A} \otimes \boldsymbol{B} \rightarrow \boldsymbol{A} / L_{1} \otimes \boldsymbol{B} / L_{2}$ of left $\boldsymbol{A} \otimes \boldsymbol{B}$-modules.

(i) There is a commutative diagram

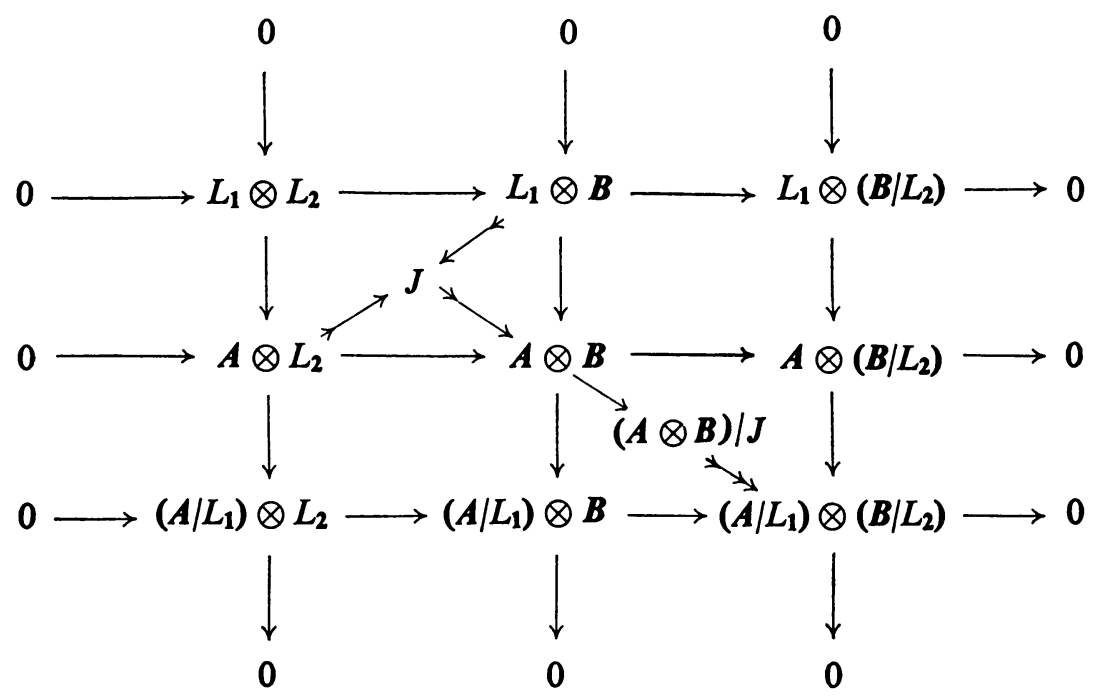

(ii) $J$ is the unique left ideal (subobject) of $\boldsymbol{A} \otimes \boldsymbol{B}$ containing $\boldsymbol{A} \otimes L_{2}$ and $L_{\mathbf{1}} \otimes \boldsymbol{B}$; furthermore $J=L$.

(iii) $A \otimes L_{2} \cap L_{1} \otimes B=L_{1} \otimes L_{2}$.

Although stated for algebras, the previous lemma is also valid for modules over a ring.

COROllary 5.4. Let $P_{1} \triangleleft A, P_{2} \triangleleft B$ be any ideals and define $P=A \otimes P_{2}$ $+P_{1} \otimes B$.

(i) Then $P \triangleleft A \otimes B$ and $A \otimes B / P \cong A / P_{1} \otimes B / P_{2}$.

(ii) If $J \triangleleft A \otimes B$ is an ideal and $J=A \otimes P_{2}+P_{1} \otimes B=A \otimes T_{2}+T_{1} \otimes B$ for some ideals $P_{1}, T_{1} \triangleleft A$ and $P_{2}, T_{2} \triangleleft B$, then $P_{1}=T_{1}$ and $P_{2}=T_{2}$.

(iii) If $P_{1}, P_{2}$ are any ideals of the form $P_{1}=L_{1}: A=\left\{a \in A \mid a A \subseteq L_{1}\right\}, P_{2}=L_{2}: B$ where $L_{1}, L_{2}$ are regular left ideals, set $L=A \otimes L_{2}+L_{1} \otimes B$. Then $P$ is an ideal with a relative right identity and $P \subseteq L:(\boldsymbol{A} \otimes \boldsymbol{B})$, where $L$ is a regular left ideal.

Proof. In this proof only " + " will mean a vector space direct sum. For some $i$, either $T_{i} \mid P_{i}$ or $P_{i} \mid T_{i} \neq \varnothing$. Suppose $\varnothing \neq T_{1}\left|P_{1}=T_{1}\right|\left(P_{1} \cap T_{1}\right)$. Choose a vector sub- 
space $\tilde{P}_{1}$ with $T_{1} \mid\left(P_{1} \cap T_{1}\right) \subseteq \tilde{P}_{1}$ and $A=P_{1}+\tilde{P}_{1}$. Then select any complementary vector subspaces $N$ and $\tilde{P}_{2}$ such that $\tilde{P}_{1}=\tilde{P}_{1} \cap T_{1}+N$ with $T_{1} \cap N=(0)$, and $\boldsymbol{B}=P_{2}+\tilde{P}_{2}$. In general, if $\boldsymbol{A}=P_{1}+\tilde{P}_{1}, \boldsymbol{B}$ are any vector spaces and the sum is direct, then $A \otimes B=P_{1} \otimes B+\tilde{P}_{1} \otimes B$ is also direct. Thus

$$
\begin{gathered}
P_{1} \otimes P_{2}+\left(\tilde{P}_{1} \cap T_{1}\right) \otimes P_{2}+N \otimes P_{2}=A \otimes P_{2}, \\
A \otimes B=P_{1} \otimes B+\left(\tilde{P}_{1} \cap T_{1}\right) \otimes P_{2}+N \otimes P_{2}+\left(\tilde{P}_{1} \cap T_{1}\right) \otimes \tilde{P}_{2}+N \otimes \tilde{P}_{2} \\
=J+\left(\tilde{P}_{1} \cap T_{1}\right) \otimes \tilde{P}_{2}+N \otimes \tilde{P}_{2} .
\end{gathered}
$$

But $(0) \neq\left(\tilde{P}_{1} \cap T_{1}\right) \otimes \tilde{P}_{2} \subseteq J$ contradicts the directness of the last sum. Thus $P_{1}=T_{1}$ and $P_{2}=T_{2}$.

Consider algebras $\boldsymbol{A}, \boldsymbol{B}$ over a field $F$, simple modules $\boldsymbol{A}-V, \boldsymbol{B}-W$ with associated division rings $D, \Delta$. Then a fundamental result of Azumaya-Nakayama says that End $V \otimes W=D \otimes \Delta$. (See [7, p. 113].) The next proposition generalizes this by not requiring that $W$ be simple by using a different proof. In the subsequent Theorem $I$ in 5.10, it will be assumed that End $V=F$ for simple modules $V$ and $W$. The next proposition then shows that in this case End $V \otimes W=$ End $W$ is a division ring.

Proposition 5.5. Suppose that $\boldsymbol{A}$ and $\boldsymbol{B}$ are algebras over a field $F$, that $V$ is $a$ simple A-module, and $W$ a cyclic $B$-module. Then

\section{End $V \otimes W=$ End $V \otimes$ End $W$}

provided either one of the following two conditions are satisfied:

(a) either $W$ is a simple $\boldsymbol{B}$-module;

(b) or $1 \in \boldsymbol{B}$.

Proof. First, clearly End $V \otimes W \supseteq$ End $V \otimes$ End $W$. Secondly, since $V$ and $W$ are modules over $\boldsymbol{A}^{\mathbf{1}}$ and $\boldsymbol{B}^{\mathbf{1}}$, it follows that $V \otimes W$ is an $\boldsymbol{A}^{\mathbf{1}} \otimes \boldsymbol{B}^{\mathbf{1}}$-module. Lastly, it will be shown that any $\boldsymbol{A} \otimes \boldsymbol{B}$-endomorphism $f$ of $V \otimes W$ commutes also with $A^{1} \otimes B^{1}$. In order to show that $f$ commutes with $A^{1} \otimes B$, by linearity it suffices to show that $f((1 \otimes b) v \otimes w)=1 \otimes b f(v \otimes w)$ for any $b \in \boldsymbol{B}, v \in V$, and $w \in W$. If assumption (a) holds, then an entirely parallel argument applies to $\boldsymbol{A} \otimes \boldsymbol{B}^{1}$, while if (b) holds, there is nothing to prove. Write $f(v \otimes w)$ as a finite sum of the form $f(v \otimes w)=\sum x d \otimes y$ with $y \in W, d \in D$, and the $x$ 's $D$-independent in $V$. Since $V$ is a simple $\boldsymbol{A}$-module, by Jacobson's density theorem, there exists an $a \in \boldsymbol{A}$ with $a v=v, a x=x$ for all $x$. Consequently

$$
\begin{aligned}
(1 \otimes b) v \otimes w & =(a \otimes b) v \otimes w, \\
f((1 \otimes b) v \otimes w) & =a \otimes b f(v \otimes w)=\sum a x d \otimes b y=1 \otimes b f(v \otimes w) .
\end{aligned}
$$

Thus $f$ commutes with $\boldsymbol{A}^{\mathbf{1}} \otimes \boldsymbol{B}+\boldsymbol{A} \otimes \boldsymbol{B}^{1}=\boldsymbol{A} \otimes \boldsymbol{B}+1 \otimes \boldsymbol{B}+\boldsymbol{A} \otimes 1$ and thus also with $A^{1} \otimes B^{1}$. Subsequently, in writing $a \otimes 1$ it is not assumed that $1 \in B$, or similarly for $1 \otimes b$ that $1 \in \boldsymbol{A}$.

Let $\{d(i) \mid i \in I\}$ be an $F$-basis of $D \equiv$ End $V$ and $\{v(j) \mid j \in J\}$ a $D$-basis of $V$. 
Let $f \in$ End $V \otimes W$ and take $v(k)$ and $w \in W$ arbitrary. Then for some finite subset $S(k, w) \subseteq I \times J$, there is a unique sum consisting of nonzero terms

$$
f(v(k) \otimes w)=\sum_{(i, j) \in S(k, w)} v(j) d(i) \otimes g(i, j, k, w)
$$

where $g(i, j, k, w) \in W$ at this point must be assumed to be a function of both $k$ and $w$. It should be noted that $\{v(i) d(j)\}$ is an $F$-basis of $V$ and that, in any tensor product of vector spaces, if the first factors belong to a basis and are distinct, then firstly, they are unique and, secondly, also the second factors $g(i, j, k, w)$ are unique (but not necessarily linearly independent). (Alternatively, the sum could be taken over all of $I \times J$ with the understanding that, for each fixed $k$ and $w$, the function $g(i, j, k, w)$ is nonzero at only a finite number of $(i, j)$.)

Let $I(k, w)$ and $J(k, w)$ be the projections of $S(k, w)$ onto $I$ and $J$. Suppose $k \notin J(k, w)$. Pick any $p \in J(k, w)$. By Jacobson's density theorem there exists an $a \in \boldsymbol{A}$ with

$$
\begin{aligned}
& a v(k)=a v(p)=v(k), \\
& a v(j)=0 \text { for } j \in J(k, w) \text { but for } j \neq p(\text { or } k) .
\end{aligned}
$$

Then

$$
\begin{aligned}
f(v(k) \otimes w) & =f((a \otimes 1) v(k) \otimes w)=a \otimes 1 f(v(k) \otimes w) \\
& =\sum_{(i, p) \in S(k, w)} v(k) d(i) \otimes g(i, p, k, w) .
\end{aligned}
$$

Since $\{v(j) d(i) \mid(i, j) \in I \times J\}$ is an $F$-basis of $V$, sums of the above forms are unique. Comparison of the latter expressions with equation (1) for $f(v(k) \otimes w)$ contradicts the assumption that $k \notin J(k, w)$.

Thus $k \in J(k, w)$. In this case there is an $a \in A$ with

$$
a v(k)=v(k), \quad a v(j)=0 \quad \text { for } j \in J(k, w) \text { but for } j \neq k .
$$

Thus $f((a \otimes 1) v(k) \otimes w)=a \otimes 1 f(v(k) \otimes w)$ shows that $J(k, w)=\{k\}$ and consequently that $S(k, w)=I(k, w) \times\{k\}$. Hence

$$
f(v(k) \otimes w)=\sum_{i \in I(k, w)} v(k) d(i) \otimes g(i, k, w)
$$

where $g(i, k, w) \equiv g(i, k, k, w)$.

For any $p \neq k$, take $a \in A$ with $a v(k)=v(p)$. By applying $a \otimes 1$ to (2) we obtain

$$
\begin{aligned}
f(v(p) \otimes w) & =f(a v(k) \otimes w)=a \otimes 1 f(v(k) \otimes w) \\
& =\sum_{i \in I(k, w)} v(p) d(i) \otimes g(i, k, w) .
\end{aligned}
$$

Substitution of $k=p$ in (2) gives

$$
f(v(p) \otimes w)=\sum_{i \in I(p, w)} v(p) d(i) \otimes g(i, p, w) .
$$


Comparison of the above two expressions shows that

$$
I(k, w)=I(p, w) \equiv I(w) \text { and } g(i, k, w)=g(i, p, w) \equiv g(i, w)
$$

are both independent of $k$. For any $w \in W$, which at this point does not yet have to be specialized to be a cyclic vector, and any $b \in \boldsymbol{B}$, use of (2) with $b w$ in place of $w$ shows that

$$
f(v(k) \otimes b w)=\sum_{i \in I(b w)} v(k) d(i) \otimes g(i, b w) .
$$

Application of $1 \otimes b$ directly to (2) gives

$$
1 \otimes b f(v(k) \otimes w)=\sum_{i \in I(w)} v(k) d(i) \otimes b g(i, w) .
$$

Again, comparison of these two expressions shows that $I(w)=I(b w)$ is independent of $w$ again and that $b g(i, w)=g(i, b w)$. It should be stressed that this independence did not use the fact that there is a cyclic vector in $W$. Thus for each $i$, the map

$$
g(i,): W \rightarrow W, \quad w \rightarrow g(i, w)
$$

is a $\boldsymbol{B}$-homomorphism and belongs to End $W$. Now take $w \in W$ to be a cyclic vector. Thus there is a finite subset $\{1, \ldots, n\} \subset I$ that indexes $d(1), \ldots, d(n)$ $\in\{d(i) \mid i \in I\}$ and $g(1),, \ldots, g(n,) \in$ End $W$ such that for any $k$ and $v(k)$, any $a \in A, b \in B$ we have

$$
f(a v(k) \otimes b w)=\sum_{i=1}^{n} a v(k) d(i) \otimes g(i, b w) .
$$

Since $V$ is simple, since $\{v(k) \mid k \in I\}$ is a $D$-basis of $V$, and since $W=\boldsymbol{B} w$, every element of $V \otimes W$ is a finite sum of elements of the form $a v(k) \otimes b w$. It follows that for any $x \in V, y \in W$ we have

$$
f(x \otimes y)=x d(1) \otimes g(1, y)+\cdots+x d(n) \otimes g(n, y),
$$

or

$$
f=d(1) \otimes g(1,)+\cdots+d(n) \otimes g(n,) .
$$

Thus End $V \otimes W=$ End $V \otimes$ End $W$.

The next lemma is well known and easy to prove.

Lemma 5.6. Suppose $N$ is any completely reducible module over an F-algebra $\boldsymbol{A}$ and $\mathscr{S}$ is a set of representatives of the simple A-modules which appear in $N$. Then $N$ is uniquely of the form $N=\oplus\{V \otimes W(V) \mid V \in \mathscr{S}\}$, where $W(V)$ is an F-vector space whose dimension is the multiplicity of $V$ in $N$, and where $a(v \otimes w)=a v \otimes w$ for $a \in A, v \in V$ and $w \in W(V)$.

The next lemma will be used several times later. In particular, it applies to a simple module. 
Lemma 5.7. Consider any ring $\boldsymbol{R}$ (or algebra) and any ideal $T \triangleleft \boldsymbol{R}$. (An identity is not assumed for $\boldsymbol{R}$ or $T$.) Suppose $N$ is any $T$-module with $N=T N$ and such that any $0 \neq z \in N$ satisfies $T z \neq(0)$. Then $N$ is in a unique natural way also an $\boldsymbol{R}$-module.

Proof. Each $x \in N$ is a finite sum $x=\sum$ ty for some $t \in T, y \in N$. For any $r \in R$, define $r x$ by $r x=\sum(r t) y$. To prove that $r x$ is well defined and unique, it suffices to show that $x=0$ implies $r x=0$. Suppose $0 \neq z \equiv \sum(r t) y$. Then $T z \neq(0)$ and for some $u \in T, 0 \neq u z=(u r) \sum t y=0$ since $u r \in T$, and this is a contradiction. Thus $N$ is an $\boldsymbol{R}$-module.

Corollary 5.8. Consider algebras $\boldsymbol{A}, \boldsymbol{B}$ over the field $F$ with $1 \notin \boldsymbol{A}$. Set $\boldsymbol{A}^{\mathbf{1}}=$ $F \times A$. Then every simple $A \otimes B$-module $N$ is also a module over $A^{1} \otimes B$, and consequently also a $B$-module.

Proposition 5.9. Consider algebras $\boldsymbol{A}, \boldsymbol{B}$ over a field $F$. Assume that

(a) Every A-module is completely reducible.

(b) For every simple A-module $V$, End $V=F$.

Then every simple $\boldsymbol{A} \otimes \boldsymbol{B}$-module is of the form $V \otimes W$, where $V, W$ are simple $\boldsymbol{A}$-, $\boldsymbol{B}$-modules; $V, W$ are unique up to $\boldsymbol{A}$-, $\boldsymbol{B}$-module isomorphisms.

Proof. Let $N$ be a simple $\boldsymbol{A} \otimes \boldsymbol{B}$-module and let $\mathscr{S}$ be any set of representatives of the classes of simple $\boldsymbol{A}$-modules, which appear in $\boldsymbol{A}-N$. Since $\boldsymbol{A} \otimes \boldsymbol{B} \triangleleft \boldsymbol{A}^{\mathbf{1}} \otimes \boldsymbol{B}^{\mathbf{1}}$, $N$ is a module over $A^{\mathbf{1}} \otimes B^{\mathbf{1}}$, and consequently also over $B \cong 1 \otimes B$ and $A \cong A \otimes 1$. By Lemma 5.6, $N$ is uniquely of the form

$$
N=\oplus\{V \otimes W(V) \mid V \in \mathscr{S}\},
$$

where $W(V)$ is an $F$-vector space whose dimension is the multiplicity of $V$ in $N$. Furthermore, $(a \otimes 1)(v \otimes w)=a v \otimes w$. By Corollary 5.8, $N$ is also a $1 \otimes \boldsymbol{B}$ $\cong \boldsymbol{B}$-module. If $b \in \boldsymbol{B}$ and $w \in W(V)$, then since $V \otimes w \cong V$ as an $\boldsymbol{A}$-module, either $(1 \otimes b) V \otimes w=0$ or $(1 \otimes b) V \otimes w \cong V$. Since $V \otimes W(V)$ is the sum of all $\boldsymbol{A} \otimes 1$ submodules of $N$ that are isomorphic to $V$, it is mapped into itself by every $\boldsymbol{A} \otimes 1$-endomorphism of $N$. Since $1 \otimes b$ is an $\boldsymbol{A} \otimes 1$-endomorphism of $V \otimes W(V)$, it follows that $(1 \otimes b) V \otimes W(V) \subseteq V \otimes W(V)$. Since $V \otimes W(V)$ is an $\boldsymbol{A} \otimes \boldsymbol{B}$-submodule of the simple module $V \otimes W$, it follows that $\mathscr{S}=\{V\}$ is a singleton; set $W=W(V)$, then $N=V \otimes W$. Clearly, $V$ is unique up to isomorphism while the $F$-dimension of $W$ is determined uniquely as the multiplicity of $V$ in $N$.

Let $b \in \boldsymbol{B}, v \in V$, and $w \in W$ be given. Write $(1 \otimes b) v \otimes w=k v \otimes \vec{w}+k(1) v(1)$ $\otimes w(1)+\cdots+k(n) v(n) \otimes w(n)$, where $n \geqq 0$; where $k, k(1), \ldots, k(n) \in F ; v, v(1)$, $\ldots, v(n)$ are $F$-independent in $V$; and $w, w(1), \ldots, w(n) \in W$. Since End $V=F$, there exists an $a \in A$ with $a v=v$ but $a v(1)=a v(2)=\cdots=a v(n)=0$. Then $(1 \otimes b) v \otimes w$ $=(a \otimes 1)(1 \otimes b) v \otimes w=k v \otimes \bar{w}$. Thus $n=0$.

If $0 \neq y \in V$ is any other element, then there is a $d \in \boldsymbol{A}$ with $d v=y$. Thus $(1 \otimes b) y \otimes w=(d \otimes 1)(1 \otimes b) v \otimes w=k y \otimes \bar{w}$. Consequently $k$ does not depend on $v$ but merely on $b$ and $w$, i.e. $k=k(b, w)$. It is easily seen that $k$ is $F$-bilinear in $b$ 
and $w$. Define $b w=k \bar{w}$. Then $W$ is a simple $\boldsymbol{B}$-module with an action compatible with $\boldsymbol{A} \otimes \boldsymbol{B}$ and $V \otimes W$, i.e. $(a \otimes b) v \otimes w=a v \otimes b w$.

Although conclusion (i) of the next theorem is already in the literature and it follows from the previously mentioned and rather complicated AzumayaNakayama theorem [7, p. 114], for the sake of completeness, a short, direct, and different proof is given below. A result in some ways related to the next theorem is the following. Consider primitive ideals $P_{1} \triangleleft A, P_{\mathbf{2}} \triangleleft B$ and the associated simple modules $V, W$ with $P_{1}=0: V=\{a \in A \mid a V=0\}, P_{2}=0: W$. It appears to be an open question whether the next theorem is true if End $V \otimes$ End $W$ is a division ring. There do exist nonsimple modules whose centralizers are division rings. The best that seems to be known in this direction is that if End $V \otimes$ End $W$ is primitive, then $A / P_{1} \otimes B / P_{2}$ is primitive [6, p. 20, Theorem 1]; the associated faithful, simple module is shown to exist, but is not concretely described. It in no way follows from either the statements of theorems nor their methods of proof in [6] that $V \otimes W$ is a simple faithful module, nor what the regular maximal left ideals that determine $A \otimes P_{2}+P_{1} \otimes B$ look like. In spite of the fact that the conclusions of the next theorem are exactly what one would naturally expect, there just does not seem to be a short proof.

5.10. Theorem IV. Consider F-algebras $\boldsymbol{A}, \boldsymbol{B}$ (where identities are not assumed) and simple modules $V=\boldsymbol{A} / L_{1}, W=\boldsymbol{B} / L_{2}$ where $L_{1}, L_{2}$ are regular maximal left ideals. Define $P_{1}=L_{1}: A=\left\{a \in A \mid a A \subseteq L_{1}\right\}, \quad P_{2}=L_{2}: B, L=A \otimes L_{2}+L_{1} \otimes B$, and $P=A$ $\otimes P_{2}+P_{1} \otimes B$. If either End $V=F$ or End $W=F$, then the following hold:

(i) $V \otimes W$ is a simple $\boldsymbol{A} \otimes \boldsymbol{B}$-module.

(ii) $V \otimes W \cong \boldsymbol{A} \otimes \boldsymbol{B} / L$; in particular $L$ is a regular maximal left ideal; furthermore $A \otimes B / P \cong A / P_{1} \otimes B / P_{2}$.

(iii) $P=L:(\boldsymbol{A} \otimes \boldsymbol{B})$; consequently $P$ is a primitive ideal of $\boldsymbol{A} \otimes \boldsymbol{B}$.

Proof. (i) Assume End $W=F$ and let $D$ be the division ring $D=$ End $V$. It suffices to show that for any $0 \neq x \in V \otimes W$ and $v \otimes w \in V \otimes W$, there exists an $a \otimes b \in \boldsymbol{A} \otimes \boldsymbol{B}$ with $a \otimes b x=v \otimes w$. Write $x$ as a finite sum $x=v(0) \otimes w(0)+\cdots$ $+v(n) \otimes w(n)$ with the $w(i)$ linearly $F$-independent and the $v(i) \neq 0$. There is a $b \in \boldsymbol{B}$ with $b w(0)=w$ and $b w(1)=\cdots=b w(n)=0$. Thus $(1 \otimes b) x=v(0) \otimes w$. Now choose an $a \in A$ such that $a v(0)=v$. Thus $a \otimes b x=v \otimes w$.

(ii) If $e(i)$ is a relative right identity for $L_{i}$, then $e(1) \otimes e(2)$ is a relative right identity for $L$. It follows from Lemma 5.3 that

$$
\begin{gathered}
(A \otimes B) / L \cong V \otimes W, \quad(A \otimes B) / P \cong A / P_{1} \otimes B / P_{2}, \\
P \subseteq 0: V \otimes W=L:(A \otimes B) .
\end{gathered}
$$

Since $V \otimes W$ is simple by (i), $L$ is a regular maximal left ideal and $0: V \otimes W$ is primitive.

(iii) In order to prove that $0: V \otimes W \subseteq P$, consider $\xi \in \boldsymbol{A} \otimes \boldsymbol{B}$ with $\xi V \otimes W=(0)$. The ideal $P_{2}$ is an intersection of regular maximal left ideals $M$ of $B$ of the form 
$M=0: w=\{b \in \boldsymbol{B} \mid b w=0\}$ for $0 \neq w \in W$ and let $M$ be any one of these. Write $A, M$, and $B$ as $F$-vector space direct sums $A=\tilde{P}_{1}+P_{1}, M=\bar{M}+P_{2}$, and $B=\bar{P}_{2}+M$ $=\bar{P}_{2}+\bar{M}+P_{2}$. Consequently $\boldsymbol{A} \otimes B=\tilde{P}_{1} \otimes \bar{P}_{2}+\tilde{P}_{1} \otimes \bar{M}+P$ is also an $F$-vector space direct sum. Choose $F$-vector space bases $\{a(\alpha)\}$ for $\tilde{P}_{\mathbf{1}},\{c(i)\}$ for $\bar{P}_{\mathbf{2}}$, and $\{b(i)\}$ for $\bar{M}$. Then $\xi$ is uniquely of the form

$$
\xi=\sum_{\alpha} \sum_{i=1}^{n} f(\alpha, i) a(\alpha) \otimes c(i)+\sum_{(\alpha, i)} f(\alpha, i) a(\alpha) \otimes b(i)+p,
$$

where $p \in P$ and $f(\alpha, i) \in F$. Note that $b(i) w \in M w=(0)$ for all $i$. Since $W=B w$, there is an element $u \in \boldsymbol{B}$ with $w=u w$. Since End $W=F$, by Jacobson's density theorem there exists for each $1 \leqq i \leqq n$ an element $b \in \boldsymbol{B}$ such that

$$
b c(i)+M=u+M, \quad b c(j) \in M \quad \text { for } j \neq i .
$$

Since $M=0: w$ and $(1 \otimes b) \xi V \otimes W=(0)$, any $v \in V$ satisfies

$$
1 \otimes b \xi v \otimes w=\sum_{\alpha} f(\alpha, i) a(\alpha) v \otimes w=0 .
$$

Thus since $v \in V$ is arbitrary, $\sum f(\alpha, i) a(\alpha) \in P_{1}=0: V$. It now follows that in the above direct decomposition of $\xi$ the first (double) sum belongs to $P_{1} \otimes B \subseteq P$ and hence is zero and must be empty. Thus

$$
\xi=\sum_{\alpha} a(\alpha) \otimes b(\alpha, i)+p, \quad b(\alpha, i)=\sum_{i} f(\alpha, i) b(i),
$$

where $a(\alpha)$ and $p \in P$ are as before and independent of $M=0: w$. In any tensor product of vector spaces, if the first factors $a(\alpha)$ are distinct and belong to a basis, then first, they are necessarily unique, and secondly, each $b(\alpha, i)$ is also unique. (However, unlike the case with the $b(i)$, now the $b(\alpha, i)$ need, of course, no longer be linearly independent.) Since corresponding to each $M=0: w$ as $w$ varies over $W$, $\xi$ has such an expression in terms of the same basis $\{a(\alpha)\}$ of $\tilde{P}_{1}$, the same expression is valid simultaneously for all $M$ and consequently each

$$
b(\alpha, i) \in \bigcap\{0: w \mid 0 \neq w \in W\}=P_{2} .
$$

Hence $\xi \in P$, and $P=0: V \otimes W=L:(A \otimes B)$.

The next corollary combines the previous theorem with Proposition 5.9 and is perhaps of more interest than the theorem. The uniqueness assertion in the corollary follows from the uniqueness in 5.9.

5.11. Corollary to Theorem IV. Consider algebras $\boldsymbol{A}$ and $\boldsymbol{B}$ over a field $F$ satisfying:

(a) Every A-module is completely reducible.

(b) End $V=F$ for every simple A-module $V$.

Then the following hold:

(i) If $V, W$ are any simple $A, B$-modules, then $V \otimes W$ is simple over $\boldsymbol{A} \otimes \boldsymbol{B}$. Conversely, every simple $\boldsymbol{A} \otimes \boldsymbol{B}$-module is uniquely of this form. 
(ii) There is a bijective map of primitive ideal spaces

$$
\begin{aligned}
\text { Prim } A \times \operatorname{Prim} B & \rightarrow \operatorname{Prim} A \otimes B, \\
\left(P_{1}, P_{2}\right) & \rightarrow A \otimes P_{2}+B \otimes P_{1} .
\end{aligned}
$$

If $1 \in A$, then, of course, the hypothesis (a) of the last corollary holds if and only if $\boldsymbol{A}$ is semisimple with the minimum condition. However, if $1 \notin \boldsymbol{A}$, there are far more general rings satisfying (a) than merely those with the minimum condition (see [1]).

6. Examples. Some examples and counterexamples are given.

The next example gives a method whereby starting from any module in the class $\mathscr{K}$, new modules in $\mathscr{K}$ are generated. Furthermore, every ring $\boldsymbol{R}$ and bimodule $\boldsymbol{R}-M-\Delta \in \mathscr{K}$ can be represented as in 6.1(a) with $V=\Delta$ and $D=\Delta$.

Example 6.1. Suppose $D-V-\Delta \in \mathscr{K}$ with $V-\Delta$ free on $\{y(\lambda) \mid \lambda \in \Lambda\}$. For any indexing set $J$ of any cardinality, define $V(\alpha)$ by $V(\alpha) \cong V$ for $\alpha \in J$ and form $M=\oplus V(\alpha) ; \Delta$ acts on $M-\Delta$ by componentwise multiplication. If $e(\alpha): V \rightarrow V(\alpha)$ $\subset M$ is the natural inclusion map, then $M-\Delta$ is free on

$$
X=\{y(\lambda) e(\alpha) \mid(\lambda, \alpha) \in \Lambda \times J\} .
$$

Any column summable matrix (with respect to $M$ as in 2.8) over $D$ whose rows and columns are indexed by $J$ acts by left multiplication on $M$. "Column finite" will refer to a finite number of nonzero entries in each column, and similarly for row finite.

(a) Let $\boldsymbol{R}$ be any subring of the ring of all column finite matrices with the property that

$$
\begin{array}{r}
\forall i, j \in J \text { and } \forall d \in D, \quad \exists\|b(\alpha, \beta)\| \in \boldsymbol{R} \text { such that } b(i, j)=d, b(\alpha, j)=0 \\
\text { if } \alpha \neq i \text { for all } \alpha \in J .
\end{array}
$$

(The latter holds, in particular, if $\boldsymbol{R}$ contains the ring of all column and row finite matrices.) Then $\boldsymbol{R}-M-\Delta \in \mathscr{K}$, and $\boldsymbol{R}-M$ is simple if and only if $D-V$ is.

(b) If $\boldsymbol{R}-M-\Delta$ is as in (a), except that now $\boldsymbol{R}$ is a subring of the ring of all row finite but column summable matrices, the same construction and conclusions as in (a) hold.

(c) The set $N$ of eventually constant vectors is a $\Delta$-submodule in $M \subset N \subset \prod V(\alpha)$, where $N$ consists of all $m \in \prod V(\alpha)$ such that all except a finite number (depending on $m$ ) of components of $m$ are constant in $V$. If $u \in N$ has each of its components equal to some fixed $y \in\{y(\lambda)\}$, then $N-\Delta$ is free on $\{y(\lambda) e(\alpha)\} \cup\{u\}$. Note that $N=M \oplus u \Delta$ as $\Delta$-modules where $u \Delta \cong V$. Take $S$ to be the set of all row finite matrices $B$ such that if a finite number (depending on $B$ ) of rows are deleted, then every column of $B$ is constant. It follows that $B$ has only a finite number of nonzero columns and that $S$ is a ring. As before, $S-N-\Delta \in \mathscr{K}$ and $S-N$ is simple if and only if $D-V$ is. 
The question arises whether in Theorem III, $\boldsymbol{A}-\boldsymbol{M}-\Delta \in \mathscr{K} F$ with $\boldsymbol{A}-\boldsymbol{M}$ faithful and cyclic implies that $\boldsymbol{A}-\boldsymbol{M}$ is simple. Aside from showing that this need not be the case, the next example also shows how nonsimple modules in the class $\mathscr{K} F$ arise naturally as quotients of rings.

EXAMPLE 6.2. Take any not necessarily commutative integral domain with $1 \in K$ that is also an algebra over a field $F$. In the $F$-algebra $A$ of all $2 \times 2$ matrices with entries in $K$, define $L$ as the left ideal of all matrices with the first column zero and set $M=A / L \cong K \oplus K$. Define $\Delta$ to be $\Delta=K$ with $\Delta$ acting on the right of $M$ by componentwise multiplication. Then $A-M-\Delta \in \mathscr{K} F, M=A(1,1)$ is a cyclic and a faithful $\boldsymbol{A}$-module, but $\boldsymbol{A}-M$ is not simple unless $K$ is a division ring.

The next example describes a method of obtaining primitive ideals of $\boldsymbol{A} \otimes \boldsymbol{B}$ not of the form $A \otimes P_{2}+P_{1} \otimes B$.

ExAmple 6.3. Take $\boldsymbol{A}=F[x]$ and $\boldsymbol{B}=F[y]$ with $x^{2}=y^{2}=0$. The local rings $\boldsymbol{A}$ and $\boldsymbol{B}$ contain unique primitive ideals $P_{1}=\boldsymbol{A} x$ and $P_{\mathbf{2}}=\boldsymbol{B} y$. Then every vector subspace $J$ with $1 \otimes 1 \notin J$ and with $x \otimes y \in J$ is an ideal. If $1 \otimes 1 \notin J$ and the codimension of $J$ is one, then $\boldsymbol{A} \otimes \boldsymbol{B} / J \cong F$ and $J$ is a maximal ideal. There are many such subspaces $J \neq A \otimes P_{2}+P_{1} \otimes B$. For example, take

$$
J=F(x \otimes y)+F(1 \otimes 1+1 \otimes y)+F(1 \otimes 1+x \otimes 1) .
$$

\section{REFERENCES}

1. J. Dauns, Chains of modules with completely reducible quotients, Pacific J. Math. 17 (1966), 235-242. MR 34 \#201.

2. - Multiplier rings and primitive ideals, Trans. Amer. Math. Soc. 145 (1969), 125-158. MR 41 \#1805.

3. - Categorical $W^{*}$-tensor product, Trans. Amer. Math. Soc. 166 (1972), 439-456.

4. K. R. Fuller, Double centralizers of injectives and projectives over Artinian rings, Illinois J. Math. 14 (1970), 658-664.

5. - On indecomposable injectives over artinian rings, Pacific J. Math. 29 (1969), 115135. MR 40 \#186.

6. M. Harada and T. Kanzaki, On Kronecker products of primitive algebras, J. Inst. Polytech. Osaka City Univ. Ser. A 9 (1958), 19-28. MR 20 \#7045.

7. N. Jacobson, Structure of rings, rev. ed., Amer. Math. Soc. Colloq. Publ., vol. 37, Amer. Math. Soc., Providence, R. I., 1964. MR 36 \#5158.

8. K. Morita, On algebras for which every faithful representation is its own second commutator, Math. Z. 69 (1958), 429-434. MR 23 \#A3788.

Department of Mathematics, Tulane University, New Orleans, Louisiana 70118 